\title{
Spirulina maxima and its effect on antioxidant activity in fructose induced oxidative stress with histopathological observations
}

Original research article/Review

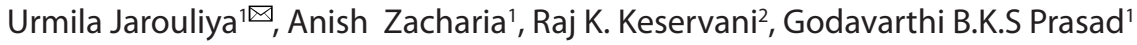

\author{
${ }^{1}$ Jiwaji University, School of Studies \\ in Biotechnology, Gwalior (M.P), India. \\ ${ }^{2}$ Rajiv Gandhi Proudyogiki Vishwavidyalaya, \\ School of Pharmaceutical Sciences, Bhopal (M.P)
}

Received August19, 2015, accepted October 29, 2015

Abstract Diabetes mellitus is a metabolic disorder characterised by hyperglycemia and oxidative stress. The aim of the present study is to explore the antioxidant effect of Spirulina maxima in rat model along with the histopathological observations. Diabetes was induced by feeding $10 \%$ fructose solution orally to Wistar rats $(n=6)$ for 30 days, analysed for plasma blood glucose and the markers of the oxidative stress [catalase (CAT), superoxide dismutase (SOD), reduced glutathione (GSH) and thiobarbituric acid reactive substances (TBARS)]. These biochemical studies were associated with histopathological examination of liver and kidney sections. The microalga Spirulina maxima being rich in proteins and other essential nutrients is widely used as a food supplement. S. maxima at a dose of 5 and $10 \%$ per $\mathrm{kg}$ and the metformin $(500 \mathrm{mg} / \mathrm{kg})$ as reference drug were given orally for 30 days to the diabetic rats. Diabetic rats showed significant $(p<0.001)$ elevations in plasma blood glucose, thiobarbituric acid-reactive substances and significant reduction in catalase, superoxide dismutase and reduced glutathione activity. Oral administration of 5 and $10 \%$ aqueous extract of S. maxima for 30 days restored not only of blood glucose levels but also markers of oxidative stress. Histopathological observations of tissues manifested that the S. maxima administration had the protective and therapeutic effects against fructose-induced abnormalities in diabetic rats. It is concluded that $S$. maxima is effective in reinstating the antioxidant activity in addition to its antidiabetic effect in type 2 diabetic rats.

Keywords Antioxidant - Diabetes - Oxidative stress - Spirulina maxima

\section{INTRODUCTION}

Diabetes mellitus is a group of metabolic disorder in which a person has high blood sugar that is hyperglycaemia or insulin resistance characterised by reducing insulin secretion (American Diabetes Association 2013). The long-term chronic hyperglycaemia is cognated with damage, dysfunction and failure of different organs with generation of oxidative stress. Oxidative stress results due to the increased production of free radicals and the reduction in antioxidant defence mechanism. In the internal environment of cells infectious diseases, toxins and nutritional imbalance form the reactive oxygen and nitrogen species. These reactive molecules in excess damage the protein, lipids and DNA (Naviaux 2012) that is associated with complications in type 2 diabetic patients.

Recent studies by researchers reported that long-term fructose feeding promotes oxidative damage and exerts detrimental effects by reducing antioxidant defences, and increasing generation of free radicals. Abnormally high levels of free radicals that cause membrane damage due to peroxidation of membrane lipids and protein glycation and the simultaneous decline of antioxidant defence mechanisms lead to cell and tissue damage (Maritim et al. 2003; Tang et al. 2006).

The oral hypoglycaemic agents currently used in clinical practice have characteristic profiles of serious side effects (Prout et al. 1974; Holman and Turner 1991; Williams and Pickup 1991). Hence, there is a need to search for newer antidiabetic agents that retain therapeutic efficacy and are devoid of side effects. Spirulina maxima is a helical microscopic cyanobacteria and blue-green aquatic plant. It appears to be a good source of organic nutrition, especially plant protein. These blue-green algae occurs naturally in alkaline water and is a complete food because of the presence of nutrients that include $\beta$-complex, vitamins, minerals, proteins, $\gamma$-linoleic acid, nutraceutical pigments (Keservani et al. 2015) and the

*E-mail: urmi_gudia@rediffmail.com

๑ Acta Facultatis Pharmaceuticae Universitatis Comenianae 
$\beta$-carotene as well as the trace elements that make it helpful for the treatment of various diseases. Some therapeutic properties, such as hypocholesterolemic effect (Nakaya et al. 1998), antioxidant and immunomodulatory activity (Kulshreshtha et al 2008), anti-herpes simplex virus activity (Hayashi 1993), body weight reduction in obese patients and antimutagenic potential, anticoagulative and antithrombotic activity (Kamble et al. 2013) and the arsenic-induced toxicity (Kulshreshtha et al. 2014) and other pharmacological properties have indicated an added advantage of an algalprotein diet for human and animal consumption (Belay 1997). This study was designed to investigate the protective and therapeutic effect of S. maxima extract on antioxidant enzyme status in fructose-induced diabetic rats.

\section{MATERIALS AND METHODS}

\section{Animals}

Male Rattus norvegicus (Wistar strain) aged 8-10 weeks (weighing about 180-220 g) were used and the animals were procured from Defence Research \& Development Establishment, Gwalior (M.P). All the rats were individually housed in plastic cages with stainless steel covers and maintained on a $12 \mathrm{~h}$ light/dark cycle at a room temperature of $25 \pm 2^{\circ} \mathrm{C}$. They were acclimatised to the laboratory condition for 1 week with access to standard diet and tap water ad libitum. All animals were treated according to the ethical guidelines approved by Institutional Animal Ethics Committee [(IHEC-09/2008), Jiwaji University, Gwalior].

\section{Chemicals}

All the chemicals used in this experiment were of analytical grade and purchased from E. Merck India Limited (Mumbai, India).

\section{Culture maintenance}

S. maxima axenic culture was acquired from algal laboratory of Indian Agriculture Research Institute (IARI), New Delhi and cultured in Zarrouk's medium at alkaline $\mathrm{pH}$ (8.5) under the light intensity of 2500 lux at $30^{\circ} \mathrm{C}$ temperature for $16 \mathrm{~h}$ light and $8 \mathrm{~h}$ dark period. On the $20^{\text {th }}$ day, exponential growth phase of the algae was observed, and then the cultures were harvested, filtered, collected, dried below $40^{\circ} \mathrm{C}$ for overnight and powdered by electric grinder for further use.

\section{Induction of diabetes}

The experimental animals were fed orally with $10 \%$ fructose solution for 30 days and the blood glucose were monitored at an interval of 15 days until the 60th day while various antioxidant parameters were analysed at an interval of 30 days, that is, at 0,30 and 60 th day.

\section{Experimental design}

The care and maintenance of animals were as per the approved guidelines of the 'Committee for the Purpose of Control and Supervision of Experiment on Animals' (CPCSEA, India). Fourty-two male Wistar rats were divided into the following groups having six rats in each group and treated as below:

Group I (normal control): Given only tap water and standard feed.

Group II (diabetic control): Diabetes was induced by giving $10 \%$ fructose for 30 days and no treatment was given.

Group III (diabetic treated with 5\% S.maxima extract): Rats of this group were fructose-diabetic and treated with $5 \% \mathrm{~S}$. maxima extract once daily for 30 days.

Group IV: These diabetic rats were treated with 5\% S. maxima extract once daily for 30 days along with co-administration of $10 \%$ fructose solution continuously.

Group V (diabetic treated with 10\% S. maxima extract): Rats of this group were fructose-diabetic and treated with $10 \% \mathrm{~S}$. maxima extract once daily for 30 days.

Group VI: Diabetic rats treated with $10 \%$ S. maxima aqueous extract once daily for consecutive 30 days along with the coadministration of fructose solution.

Group VII (diabetic treated with metformin): This group rats were treated with metformin $(500 \mathrm{mg} / \mathrm{kg})$ once daily for 30 consecutive days.

\section{Preparation and administration of Spirulina maxima extract}

The S. maxima aqueous extract was prepared by suspending dry powder in water at a concentration of 5 and $10 \mathrm{~g} / 100$ $\mathrm{ml}$ of water. The prepared infusion was given orally once to diabetic animals throughout the experimental period by intragastric tube (cannula).

\section{BIOCHEMICAL ANALYSIS}

\section{Blood glucose estimation}

Blood glucose was estimated spectrophotometrically by using the commercial diagnostic kits (E. Merck India Ltd, Mumbai, India) based on glucose oxidase peroxidase method (Trinder 1969).

\section{Estimation of oxidative stress markers in blood}

Before collecting the blood sample from retro orbital plexus of rat eyes, the animals were fasted for $6 \mathrm{hrs}$, anesthetised by mild ethyl ether and the blood was collected through heparinised capillary into heparinised vials once before and at 30 day intervals for biochemical analyses. Reduced glutathione $(\mathrm{GSH})$ was estimated in a whole blood $(0.2 \mathrm{ml})$ and remaining sample was used for preparation of haemolysate 
for estimation of catalase (CAT), superoxide dismutase (SOD) and thiobarbituric acid reactive substances (TBARS). All estimations were carried out immediately after collecting the samples.

Estimation of GSH level in blood was carried out by the method originally described by Ellman (Ellman 1959). TBARS were determined as an index of lipid peroxidation (LPO) (Ohkawa et al. 1979). The SOD activity was assayed by the method of Winterbourn et al (Winterbourn et al. 1975). CAT was determined by monitoring the decomposition of hydrogen peroxide as described by Sinha (Sinha 1972). Protein concentration in the haemolysate was determined according to Lowry et al method (1951), using bovine serum albumin (BSA) as standard (Lowry et al. 1951).

\section{Histopathological study}

At the end of experiment on the $61^{\text {st }}$ day, rats were fasted for overnight before sacrifice. They were anaesthetised with ethyl ether and the blood was collected by cardiac puncture. The liver and kidneys were removed, pruned, blotted and weighed and these tissues were fixed in aqueous Bouin's fluid solution. After proper fixation, small pieces were dehydrated by passing successfully through a different mixture of ethyl alcohol-water, cleaned in xylene and embedded in paraffin wax. Sections of liver and kidney (5-6 $\mu \mathrm{m}$ thick) were prepared and then stained with haematoxylin and eosin dye, which were mounted in neutral deparaffinated xylene (DPX) for microscopic observations (McManus and Mowry 1965).

\section{Statistical analysis}

The data were analysed using one-way Analysis of variance (ANOVA) (Bonferroni t-test) employing Sigma Stat, statistical software, version 1.0 (Jandal corporation, USA). P $<0.001$ and $\mathrm{P}<0.05$ were regarded as statistically significant.

\section{RESULTS}

Figure 1 shows that by giving 10\% fructose solution to Wistar rats daily for 30 days significantly $(p<0.001)$ increases the blood glucose level by $85-98 \%$ and the lipid peroxidation (TBARS) products by $70 \%$ (Figure 2) in diabetic rats when compared with normal control group rats. The activities of SOD and CAT were significantly $(p<0.001)$ reduced by 60 and $40 \%$, respectively (Figures 3 and 4). GSH level was marginally affected by $15 \%$ (Figure 5 ).

Effect of $S$. maxima aqueous extract on blood glucose level in fructose-induced diabetic rats after 30-day treatment

While given treatment to diabetic rats with 5 and $10 \%$ S. maxima extract, blood glucose levels were significantly reduced by 60 $(P<0.001)$ and $54 \%(P<0.001)$ compared with normal control rats.

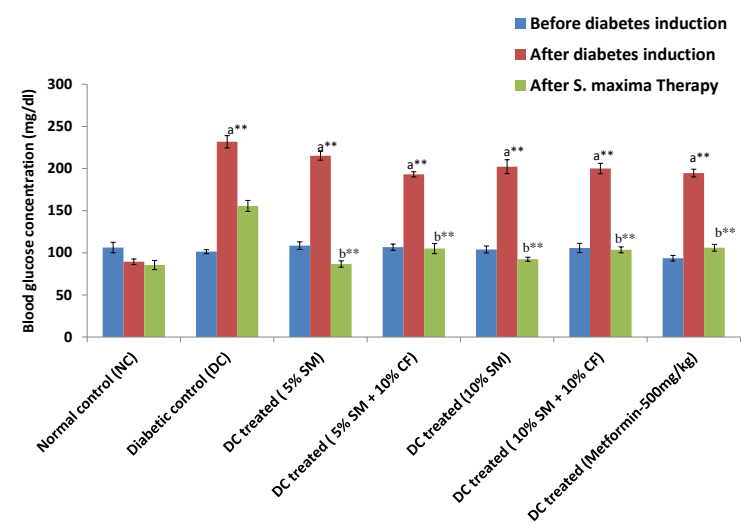

Figure 1. Effect of S. maxima administration on blood glucose level Abbreviations: SM - Spirulina maxima, CF - Continuous fructose. Units: $m g / d l$. The values expressed as mean $\pm S E, n=6$.

${ }^{a} p<0.05\left({ }^{*}\right),{ }^{a} p<0.001\left(^{* *}\right)$ significant compared with normal control group.

${ }^{b} p<0.05\left({ }^{*}\right),{ }^{b} p<0.001\left(^{* *}\right)$ significant compared with diabetic control group.

The dabetic group treated with continuous fructose feeding and S. maxima had reduced the blood glucose levels significantly by $45-46 \%$. While in the allopathic drug (metformin) treated group, reduction in the blood sugar level was observed by $45 \%$ within 30 days of the S. maxima therapy (Figure 1).

Effect of S. maxima aqueous extract on oxidative stress markers in fructose-induced diabetic rats after 30-day treatment

Thiobarbituric acid reactive substances (TBARS) level The level of elevated lipid peroxidation was considerably reduced to $60.33 \%$ ( $\mathrm{P}<0.001$ ) with $5 \%$ S. maxima and $39.25 \%$ with $10 \%$ S. maxima compared with normal control group. Whereas the diabetic group treated with S. maxima and on continual fructose feeding generated reduction in TBARS levels by $32-45 \%$. In the metformin $(500 \mathrm{mg} / \mathrm{kg}$ ) treated diabetic group, there is reduction in TBARS levels by $29.64 \%$ within 30 days of therapy (Figure 2).

\section{SOD activity}

The administration of 5 and $10 \%$ S. maxima to diabetic rats increased the SOD activity by $190(P<0.001)$ and 230\% ( $P$ $<0.001$ ) after 30 days of the therapy. The S. maxima-treated and constant fructose-fed diabetic rats had elevation of 185$250 \%(P<0.001)$ in the SOD activity. Whereas the metformin $(500 \mathrm{mg} / \mathrm{kg})$ treated diabetic rats had marginal change in SOD activity (7\%) (Figure 3).

\section{Catalase activity}

The administration of 5 and $10 \%$ S. maxima to diabetic rats increased the CAT activity by $70.64(P<0.05)$ and $96.29 \%$ $(\mathrm{P}<0.001)$ after 30 days of the therapy. The diabetic group 


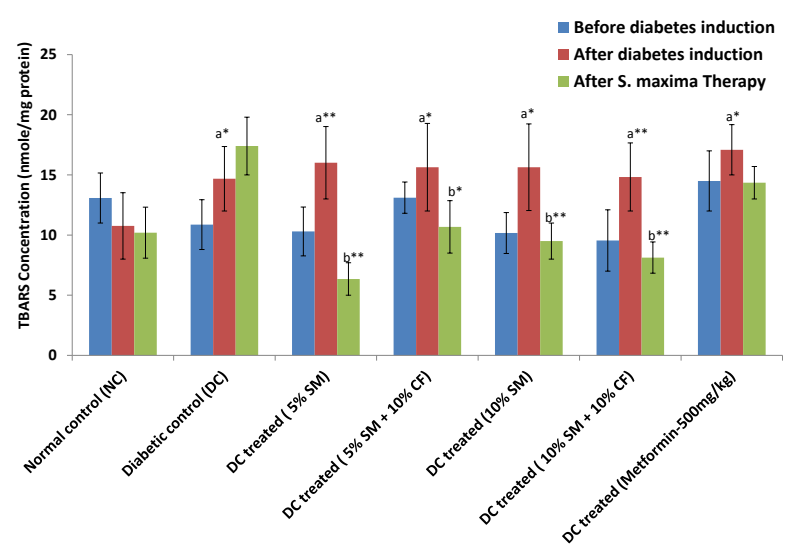

Figure 2. Effect of S. maxima administration on TBARS level Abbreviations: SM - Spirulina maxima, CF - Continuous fructose. Units: $n$ mole/mg protein. The values expressed as mean $\pm S E, n=6$. ${ }^{a} p<0.05(*),{ }^{a} p<0.001\left(^{* *}\right)$ significant compared with normal control group.

$\left.{ }^{b} p<0.05\left({ }^{*}\right),{ }^{b} p<0.0011^{* *}\right)$ significant compared with diabetic control group.

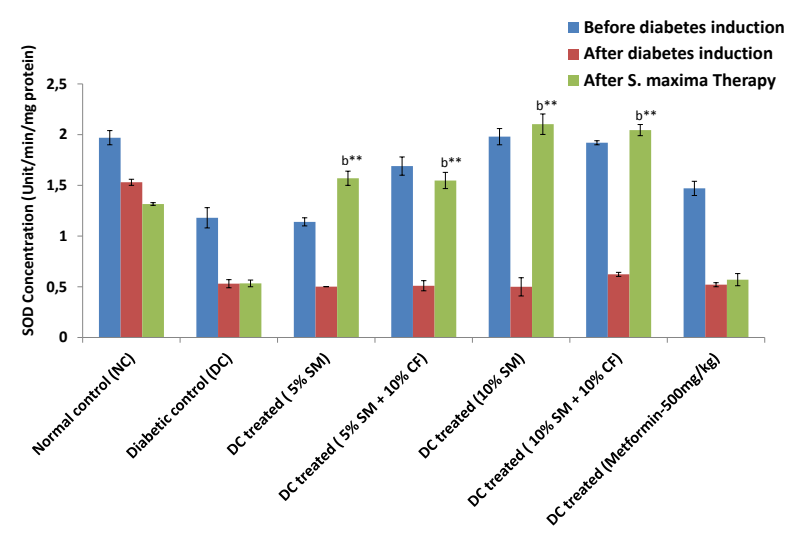

Figure 3: Effect of S. maxima administration on SOD activity Abbreviations: SM - Spirulina maxima, CF-Continuous fructose. Units: Unit/min/mg protein. The values expressed as mean $\pm S E$, $n=6$.

${ }^{a} p<0.05\left({ }^{*}\right),{ }^{a} p<0.001\left({ }^{* *}\right)$ significant compared with normal control group.

$\left.{ }^{b} \mathrm{p}<0.05\left(^{*}\right),{ }^{b} \mathrm{p}<0.001{ }^{* *}\right)$ significant compared with diabetic control group.

explored with S. maxima and ongoing fructose feeding increased the CAT activity by $41-49 \%(P<0.05)$ and the CAT activity in metformin $(500 \mathrm{mg} / \mathrm{kg})$ treated diabetic rats were increased by $22 \%$ (Figure 4).

\section{Reduced GSH level}

The results in Figure 5 showed that the GSH level was found to be elevated by $21 \%(P<0.05)$ and $9 \%$ with 5 and $10 \% S$. maxima, respectively, in the diabetic rats after 30 days of the

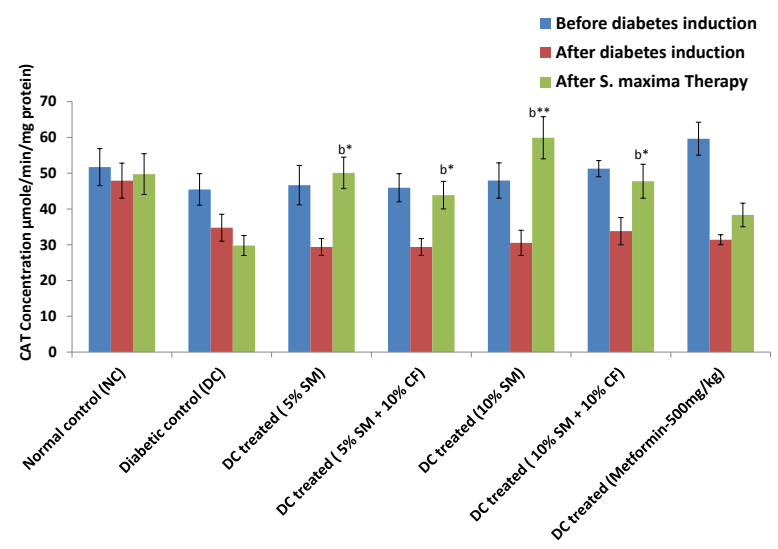

Figure 4. Effect of S. maxima administration on CAT activity Abbreviations: SM - Spirulina maxima, CF-Continuous fructose. Units: $\mu \mathrm{mole} / \mathrm{min} / \mathrm{mg}$ protein. The values expressed as mean $\pm S E, n=6$. ${ }^{a} p<0.05\left(^{*}\right),{ }^{a} p<0.001\left(^{* *}\right)$ significant compared with normal control group.

$\left.{ }^{b} p<0.05\left(^{*}\right),{ }^{b} p<0.0011^{* *}\right)$ significant compared with diabetic control group.

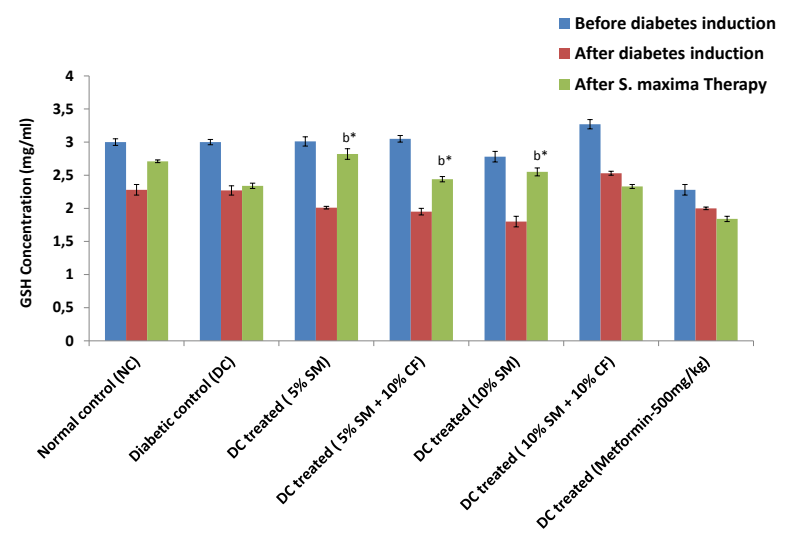

Figure 5. Effect of S. maxima administration on reduced GSH level Abbreviations: SM - Spirulina maxima, CF-Continuous fructose. Units: $\mathrm{mg} / \mathrm{ml}$. The values expressed as mean $\pm S E, n=6$.

${ }^{a} p<0.05\left({ }^{*}\right),{ }^{a} p<0.001\left(^{* *}\right)$ significant compared with normal control group.

$\left.{ }^{b} p<0.05\left({ }^{*}\right),{ }^{b} p<0.001{ }^{* *}\right)$ significant compared with diabetic control group.

therapy. There was a marginal change in GSH level by $4 \%$ in the S. maxima and $10 \%$ continuous fructose-fed diabetic rats. While the metformin $(500 \mathrm{mg} / \mathrm{kg})$ treatment had marginal changes in blood GSH level by $8 \%$ after 30 days of therapy (Figure 5).

\section{Histopathological observations}

\section{Liver histopathology}

Liver histology of control rats showed normal hepatic cord pattern, hepatic lobules and hepatocytes with normal 

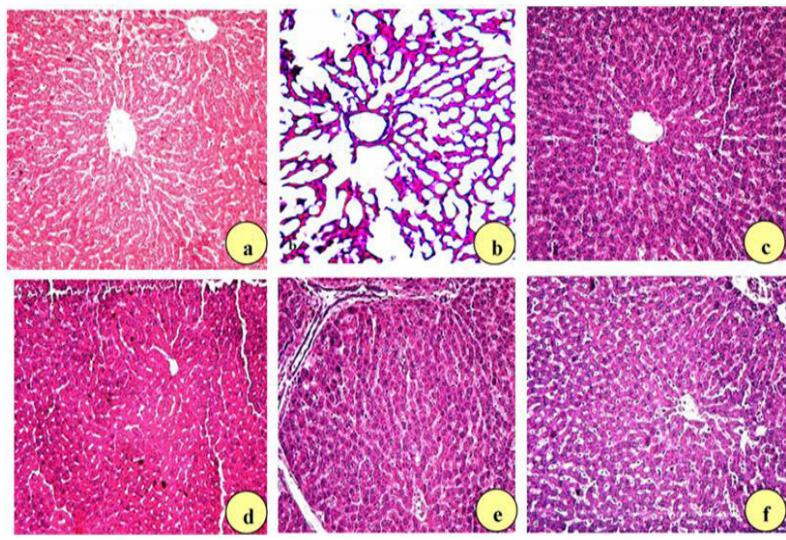

Figure 6. (a-f): Effect of S. maxima administration on liver histology of rats (haematoxylin and eosin $\times 100$ )

a. Normal control liver

b. Diabetic control liver

c. Diabetic liver treated with 5\% S. maxima

d. Diabetic liver treated with 5\% S. maxima along with $10 \%$ continuous fructose

e. Diabetic liver treated with $10 \%$ S. maxima

f. Diabetic liver treated with $10 \%$ S. maxima along with $10 \%$ continuous fructose
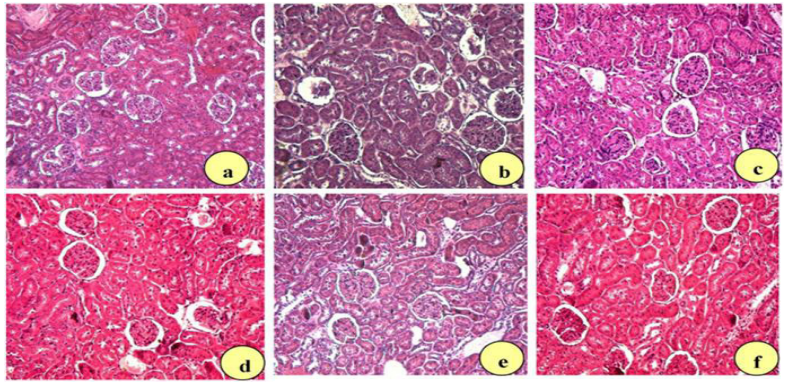

Figure $7(a-f)$. Effect of S. maxima administration on kidney histology of rats (haematoxylin and eosin $\times 100$ ).

a. Normal control kidney

b. Diabetic control kidney

c. Diabetic kidney treated with 5\% S. maxima

d. Diabetic kidney treated with 5\% S. maxima along with $10 \%$ continuous fructose

e. Diabetic kidney treated with $10 \%$ S. maxima

f. Diabetic kidney treated with $10 \%$ S. maxima along with $10 \%$ continuous fructose

architecture (Figure 6a). Liver histology of diabetic rats showed congestion of blood sinusoids around the central vein with sporadic haemorrhage and the large number of hepatocytes were pyknotic in appearance, deposition of exudates in the portal tract with signs of necrotic hepatocytes (Figure $6 \mathrm{~b}$ ). The hepatic lesions were minimal in rat liver treated with S. maxima alone. However, few inflammatory cells were observed in the vicinity of central canal. The general hepatic architecture was normal (Figure $6 c$ and e). It is observed that the severity of degenerative changes in the liver of rats co-administered with fructose and S. maxima were reduced significantly as compared with the diabetic rats (Figure 6d and f).

\section{Renal histopathology}

Renal histology of control rats showed normal packed malpighian corpuscles, renal tubules and blood vessel which were supported by scanty reticular fibre (Figure 7a). Histological examination of kidneys of diabetic rats showed multiple areas of tubular vacuolations with parenchymal hypertrophy. Renal tubules also revealed degenerative changes in the epithelium characterised by eosinophilic appearance with pyknotic nuclei (Figure 7b) Renal histology of diabetic rats treated with S. maxima alone showed minimal lesions and renal histology appeared normal (Figure $7 c$ and e). These vacuolative degenerative changes were also significantly reduced in the renal sections of rats coadministered with fructose and S. maxima (Figure $7 d$ and f).

\section{DISCUSSION}

Recently, much attention has been focussed on the role of oxidative stress and it has been suggested that oxidative stress may be the key and common factor in the pathogenesis of different diabetic complications (Sepici-Dincel et al. 2007). The mechanisms that contribute to increased oxidative stress in diabetes include non-enzymatic glycosylation, autooxidative glycosylation and metabolic stress. Diabetes and experimental animal models exhibit high oxidative stress due to persistent and chronic hyperglycemia, which depletes the activity of antioxidative defence system and thus, prevents the de novo free radicals elimination/neutralisation (Kamalakanan and Prince 2006). Oxygen-free radicals react with all biological substances; however, the most susceptible ones are polyunsaturated fatty acids. Reactions with these cell membranes constituents lead to lipid peroxidation (LPO). These elevated levels of LPO disrupt the membrane transport and signalling function (Arulselvan and Subramanian 2007). Thus, this study exhibits a potent anti-oxidant function of S. maxima against fructose-induced oxidative stress. In a preliminary study done by me to see the hyperglycemic and hyperlipidemic effect in fructose-fed Wistar rats, the findings suggested that the S. maxima administration results the anti-hyperglycemic and anti-lipidemic along with the hepatoprotective activity (Jarouliya et al. 2012). This finding confers that by inducing diabetes through fructose feeding to Wistar rats for the period of 30 days resulted in a significant elevation in TBARS levels with simultaneous reductions in GSH, SOD and CAT activity. As shown in Figure 2, the fructosefed diabetic rats with elevated level of TBARS in the blood may be due to high level of circulating glucose, which, in turn, increase free radicals production, glucose auto-oxidation and glycation (oxidative modification) of protein (Wolf et al. 1990). The present study shows that the administration of S. maxima significantly decreased the blood TBARS level (Figure 2). 
In this study, the administration of S. maxima extracts to fructose-fed rats significantly increased the SOD and CAT activities in blood (Figures 3 and 4). The SOD and CAT are the two major scavenging enzymes that remove toxic-free radicals in vivo. A decrease in the activity of these antioxidants can lead to an excess availability of superoxide anion $\mathrm{O}^{2-}$ and hydrogen peroxide in biological systems, which, in turn, generate hydroxyl radicals, resulting in initiation and propagation of lipid peroxidation. The elevated activities of SOD and CAT suggest that S. maxima have antioxidant property, which shows the beneficial effect against pathological complications caused by the presence of free radicals (Aebi 1984). Also, the S. maxima therapy elevated the GSH level in fructose-induced diabetic rats. The increased GSH level protects from the toxic effects of reactive oxygen species or peroxidative damages and plays an important role in the removal of peroxides and foreign compounds (Shan and Jones 1990). S. maxima is found to significantly increase the GSH level in blood (Figure 5), it may be because of the activation of GSH synthetase that increases the biosynthesis of GSH by S. maxima extract administration. Histopathological observations of tissues (liver and kidney) revealed that the $S$. maxima administration had the protective and therapeutic effects against fructose-induced abnormalities in male Wistar rats (Figures 6 and 7]. This study shows that the administration of aqueous extract of S. maxima decreased TBARS level with concurrent increase in key antioxidants SOD, CAT and
GSH levels in blood. The antioxidant effects of S. maxima may be attributed to $\beta$-carotene, tocopherol, and phenolic compounds working individually or in synergy (Miranda et al. 1998; Hsiao et al. 2005; Nagakao et al. 2005).

\section{CONCLUSIONS}

The present investigation shows that the oral administration of 5 or $10 \%$ aqueous extract of S. maxima for 30 days restored significantly not only the blood glucose levels but also markers of oxidative stress. The activities of antioxidant enzymes (TBARS, SOD, CAT and GSH) in diabetic rats were reverted back to near-normal level after treatment with 5 or $10 \%$ S. maxima extract. The histopathological studies also revealed the therapeutic effects of S. maxima extract on liver and kidney tissues. The effect of 5\% S. maxima extract was more pronounced than $10 \%$ extract.

\section{ACKNOWLEDGEMENT}

We are thankful to UGC, New-Delhi and the Madhya Pradesh Council of Science and Technology, Bhopal (MPCOST), India for providing the research grant.

\section{CONFLICT OF INTEREST}

Authors declare no conflict of interest.

\section{References}

[1] Aebi H. Catalase in vitro. In: Colowick S.P., Kalpan, N.O (Eds), Methods in Enzymology, Academic press, 1984, 121-126.

[2] American Diabetes Association. Diagnosis and Classification of Diabetes Mellitus. Diabetes Care, Volume 36, Supplement 1, January 2013, S67-S74.

[3] Arulselvan P, Subramanian SP. Beneficial effects of Murraya Koenigii leaves on antioxidant defense system and ultra structural changes of pancreatic $\beta$-cells in experimental diabetes in rats. Chem Biol Interact. 2007, 165: 155-164.

[4] Belay A. Mass culture of Spirulina outdoors-The Earthrise Farms experience. In: Vonshak A., ed. Spirulina platensis (Arthrospira), Physiology, cell-biology and biotechnology, Taylor and Francis, London, 1997, 131-158.

[5] Ellman GL. Tissue sulfhydryl groups. Arch Biochem. 1959, 82: 7077.

[6] Hayashi K. An extract from Spirulina platensis in a selective inhibitor of herpes simplex virus type 1 penetration into cells. Phytoth Res. 1993, 7: 76-80.

[7] Holman RR, Turner RC. Oral agents and insulin in the treatment of NIDDM. In: pickup JC, Williams G (eds), Text book of Diabetes, Blackwell, Oxford, 1991, 467-469.

[8] Hsiao G, Chou PH, Shen MY, Chou DS, Lin CH, Sheu JR. C-phycocyanin, a very potent and novel platelet aggregation inhibitor from Spirulina platensis. J Agricul Food Chem. 2005, 20; 7734-7740.
[9] Jarouliya U, Zacharia J.A, Kumar P, Bisen PS, Prasad GB. Alleviation of metabolic abnormalities induced by excessive fructose administration in Wistar rats by Spirulina maxima. Indian J Med Res. 2012, 135: 422-428.

[10] Kamalakanan N, Prince PSM. Antihyperglycemic and antioxidant effect of rutin, a polyphenolic flavonoid, in streptozotacininduced diabetic wistar rats. Basic and Clinical Pharmacol Toxicol. 2006, 98: 97-103.

[11] Kamble SP, Gaikar RB, Padalia RB, Shinde KD. Extraction and purification of C-phycocyanin from dry Spirulina powder and evaluating its antioxidant, anticoagulation and prevention of DNA damage activity. J Appl Pharmceut Sci. 2013, 8: 149-153.

[12] Keservani RK, Kesharwani RK, Sharma AK, Jarouliya U. Dietary Supplements, Nutraceutical, and Functional Foods in Immune Response (Immunomodulators), In: Nutraceutical and Functional Foods in Human Life and Disease Prevention. Edited by Debasis Bagchi, Anand Swaroop, Harry G. Preuss. CRC Press, Taylor and Francis, Boca Raton, Fl. Chapter 20, 343-360, 2015. ISBN: 9781482237214 (In Press).

[13] Kulshreshtha A, Jarouliya U, Prasad GBKS, Flora SJS, Bisen PS. Arsenic-induced abnormalities in glucose metabolism: Biochemical basis and potential therapeutic and nutritional interventions. World J TransI Med. 2014, 3: 96-111. 
[14] Kulshreshtha A, Zacharia A, Jarouliya U, Bhadauriya P, Prasad GBKS, Bisen PS. Spirulina in health care management, Curr Pharmaceut Biotechnol. 2008, 9: 400-405.

[15] Lowry OH, Roesborough MJ, Farr AL, Randall KJ. Protein measurement with folin phenol reagent. J Biol Chem. 1951, 193: 265-275.

[16] Maritim AC, Sanders RA, Watkins JB. Diabetes, oxidative stress, and antioxidants: a review. J Biochem Mol Toxicol. 2003, 17: 2439.

[17] Mc Manus JFA, Mowry RW. General methods for study of the cell and its structure, In staining methods: Histologic and Histochemical. Harper, Newyork, 1965, 73-90.

[18] Miranda MS, Cintra RG, Barros S.B.M., Mancini F.J. Antioxidant activity of the micro alga Spirulina maxima. Braz J Med Biol Res. 1998, 31; 1075-1079.

[19] Nagakao S, Shimizu K, Kaneko $H$ et al. A novel protein C-phycocyanin plays a crucial role in the hypocholesterolemic action of Spirulina platensis concentrate in rats. J Nutr. 2005, 135: 2425-2430.

[20] Nakaya N, Homma Y, Goto Y. Cholesterol lowering effects of Spirulina. Nutr Reports Int. 1998, 37: 1329-1337.

[21] Naviaux, RK. Oxidative Shielding or Oxidative Stress? J Pharmacol Exp Ther. 2012, 342:608-618.

[22] Ohkawa H, Ohishi N, Yagi K. Assay for lipid peroxides in animal tissues by thiobarbituric acid reaction. Anal Biochem. 1979, 95: 351-358
[23] Prout TE, Malassie WJ, Pirart J. Proceedings VIII Congress of International Diabetes Federation, Exceptra Medica, Amsterdam, $1974,162$.

[24] Sepici-Dincel A, Acikgoz S, Cevik C, Sengelen M, Yesilada E. Effects of in vivo antioxidant enzyme activities of myrtle oil in normoglycemic and alloxan diabetic rabbits. J Ethnopharmacol. 2007, 110: 498-503.

[25] Shan XQ, Jones DP. Glutathione dependent protection against oxidative injury, Pharmacol. Therapeut. 1990, 47: 61-71.

[26] Sinha A.K. Colorimetric assay of catalase. Anal Biochem 1972; 47: 389-394.

[27] Tang L, Wei W, Chen L, Liu S. Effects of berberine on diabetes induced by alloxan and a high-fat/high-cholesterol diet in rats. J Ethanopharmacol. 2006, 108: 109-115.

[28] Trinder P. Determination of glucose in blood using glucose oxidase with an alternative oxygen acceptor. Anal Clinical Biochem. 1969, 6: 24-27.

[29] Williams G, Pickup J. New drugs in the management of diabetes mellitus. In Pickup JC, Williams G (eds). Textbook of Diabetes II, Blackwell, Oxford, 1991, 977-993.

[30] Winterbourn CC, Hawkins RE, Carrell BM. Estimation of red cell superoxide dismutase activity. J. Lab Clinic Med. 1975, 337-341.

[31] Wolf SP, Jian ZY, Hunt JV. Protein glycation and oxidative stress in diabetes mellitus and aging. Free Radical Biol Med. 1990, 10: 339-352. 